Ion activation energy delivered to wounds by atmospheric pressure dielectric-barrier discharges: sputtering of lipid-like surfaces

This article has been downloaded from IOPscience. Please scroll down to see the full text article.

2012 J. Phys. D: Appl. Phys. 45115203

(http://iopscience.iop.org/0022-3727/45/11/115203)

View the table of contents for this issue, or go to the journal homepage for more

Download details:

IP Address: 141.211.173.82

The article was downloaded on 26/06/2013 at 15:43

Please note that terms and conditions apply. 


\title{
Ion activation energy delivered to wounds by atmospheric pressure dielectric-barrier discharges: sputtering of lipid-like surfaces
}

\author{
Natalia Yu Babaeva ${ }^{1}$, Ning Ning ${ }^{2}$, David B Graves ${ }^{2}$ and Mark J Kushner ${ }^{1,3}$ \\ ${ }^{1}$ University of Michigan, Department of Electrical Engineering and Computer Science, 1301 Beal Ave., \\ Ann Arbor, MI 48109-2122, USA \\ ${ }^{2}$ University of California, Department of Chemical and Biomolecular Engineering, 201 Gilman Hall, \\ Berkeley, CA 94720-1462, USA \\ E-mail: nbabaeva@umich.edu,mjkush@umich.edu,ningning@berkeley.edu and graves@ berkeley.edu
}

Received 9 December 2011, in final form 13 February 2012

Published 5 March 2012

Online at stacks.iop.org/JPhysD/45/115203

\begin{abstract}
The application of atmospheric pressure plasmas to human tissue has been shown to have therapeutic effects for wound healing and in treatment of skin diseases. These effects are attributed to production of UV photon fluxes, electric fields and beneficial radicals which intersect with biological reaction chains, and to energetic ions bombarding the surface. In this paper we report on results from a computational investigation of the ion energy and angular distributions (IEADs) in a dielectric-barrier discharge sustained in air incident directly on cell membranes for small dry and wet wounds in human skin. We found that ion energies in excess of 20-30 eV can be delivered onto cell membranes of dry wounds, and up to $60 \mathrm{eV}$ onto the liquid interface of the wet wound. The details of the IEADs depend on the orientation of the cell membrane and on the relative location of the plasma streamer to the wound. Using results from a molecular dynamics simulation of ion sputter probabilities of typical lipid-like material, we show that prolonged exposure of the cell membrane to such IEADs can produce significant carbon removal.
\end{abstract}

(Some figures may appear in colour only in the online journal)

\section{Introduction}

Cold atmospheric pressure plasma (CAPP) treatment of living tissue is being used in a variety of therapies now referred to as plasma medicine [1-5]. Some examples of medical applications of CAPP are the use of plasmas in the treatment of dental cavities [6], sterilization of surfaces [7], and treatment of skin diseases $[8,9]$ and melanoma $[3,10]$. In particular, CAPP has been shown to have therapeutic effects in wound healing [11]. The direct application of plasma triggers a sequence of biological responses in tissues and cells which speeds the recovery of ulcerated skin and wounds. This is partly due to the high bactericidal effectiveness of plasmas

\footnotetext{
3 Author to whom any correspondence should be addressed.
}

while not harming mammalian cells. CAPP produces a unique environment of reactive oxygen species (ROS) and reactive nitrogen species (RNS), charged particles, photons, heat, and electrostatic and electromagnetic fields, many of which are known to induce biological effects [12]. For example, nitric oxides (NO) can promote cell proliferation while hydroxyl radicals $(\mathrm{OH})$ are anti-bacterial agents $[8,9,13]$, as are UV photons [1,14]. An issue of current research is the manner of penetration of plasma produced species into tissue or the biological fluid which covers most wounds and tissue. For example, the maximum penetration depth of plasma produced ROS/RNS into biological fluids prior to reacting may be at most a few tens of microns. The penetration into the skin or living tissue may be even more limited [15]. 
The role of kinetically energetic particles, such as ions produced by the plasma, in these processes is even less clear. It is well known that ions having energies of many $\mathrm{keV}$ are effective at sputtering cell membranes and biological tissue [16]. In prior studies [17] we found that dielectric-barrier discharges (DBDs) sustained in atmospheric pressure air, a common plasma source used in plasma medicine [7], are able to deliver pulses of energetic ions to dielectric surfaces lasting up to a few ns. Depending on the voltage and dielectric constant of the underlying material, ion energies in excess of tens of eV can be delivered to the top surface, accounting for a few percent of incident ions averaged over the pulse. In an analogous manner, it is likely that DBDs incident onto living tissue and biological fluids also deliver bursts of energetic ions. The details of the interaction of such low-energy ions with biological tissue and fluids are not clearly known. However, given that the energy of these ions can exceed the bond and dissociation energies of the molecules in biological fluids and on the surface of cells, it is likely that some bond breaking occurs, as quantitatively observed in low pressure microwave plasma exposure of lipid layers [18]. As such, ion bombardment may produce ROS/RNS within the fluid or on the surface of cells, or activate the surface to enable otherwise endothermic processes to occur in analogy to activation of etching and deposition processes in semiconductor processing [19]. Therefore, the therapeutic effects of CAPP may also be partially attributed to energetic ions impinging onto wounds and tissue surfaces $[1,7]$.

In this paper, we discuss one of the least investigated sources of activation energy in the CAPP treatment of living tissue and wounds-pulses of energetic ions produced by DBDs. We computationally investigated ion energy and angular distributions (IEADs) incident onto a small wound in human skin, and onto the surface of a liquid filled wound. Using molecular dynamics (MD) simulations of lipid-like layers that have some of the properties of cell membranes, we predict the carbon sputter probabilities by low-energy ion bombardment $(<150 \mathrm{eV})$. Using the computed IEADs and these probabilities, we comment on the possible effects of CAPP exposure of these cell membranes.

We found that ion energies in excess of $20-30 \mathrm{eV}$ can be delivered within the small wound depending on the location of the filament relative to the wound. Ion energies up to $60 \mathrm{eV}$ can be incident onto the surface of a liquid filled wound, higher average energies than onto the dry wound due to the larger dielectric constant of the fluid compared to the bare cells. It is well known that ions play an important role in plasma-treated liquids (such as blood serum) as catalysts and in triggering biological responses. Although the physicalchemical interaction of these ions with the liquid covering the wound is a critically important issue, the mechanisms are poorly understood, and their discussion is beyond the scope of this particular investigation.

Results from the MD simulations indicate that carbon atom sputtering yields from lipid-like films following argon ion bombardment range from 1.0 for energies of $30-40 \mathrm{eV}$ to in excess of 10 for ion energies in excess of $100 \mathrm{eV}$. When convolved with the predicted IEADs, effective sputtering yields in excess of 0.1 are produced. We note that the lipidlike films used in these MD simulations are not intended to represent the lipid bi-layers of cell membranes, but in the absence of better models of the tissue material interacting with CAPP, we have used these films as approximate models.

The models used in this investigation are described in sections 2 and 3. We quantify IEADs delivered to dry wounds resulting from DBD filaments arriving directly on and adjacent to the wounds in section 4. IEADs incident onto the surface of a wound filled with blood serum are also discussed in section 4. Results from the MD simulations are in section 5, and a discussion of the implications of the MD simulations on DBD treatment of cells is in section 6. Our concluding remarks are in section 7 .

\section{Description of the plasma model}

To provide insights into the IEADs delivered by filaments in DBDs intersecting with wounded human skin, we computationally investigated the dynamics of a single plasma filament at different locations near the wound. The model, nonPDPSIM is the same used in [17,20]. nonPDPSIM is a two-dimensional simulation implemented on an unstructured numerical mesh in which Poisson's equation and transport equations for charged and neutral species are solved. Poisson's equation is solved throughout the entire computational domain (except in metals where the potential is specified as a boundary condition). Continuity equations for gas phase charged and neutral particles are only solved in the plasma region. Conservation equations for surface and volume charge are solved on and inside all non-metallic materials.

An unstructured mesh having a dynamic range of $>1000$ is used to enable both the DBD reactor scale and the sheath at the surface of the skin, as well as cellular structure within the wound to be resolved in a single mesh [20]. The unstructured numerical mesh has triangular elements with refinement regions. The meshes consist of approximately 10000-12000 nodes, of which about 4000-6000 are in the plasma region. The plasma is sustained in 1 atm of dry air. The reaction mechanism is discussed in [17]. Note that typical experiments on plasma tissue interactions are often conducted in humid air, and so our calculation is idealized in this regard. In discharges sustained in humid air, in addition to nitrogen and oxygen ions, water ions and water-cluster ions are formed. The fraction of water ions that are initially produced during the current pulse is roughly proportional to the mole fraction of the water vapour, at most a few percent. During the short current pulse ( $<$ a few ns), there is insufficient time for the series of charge exchange reactions to occur that produce water-cluster ions. Therefore, even in humid air, the majority of ions incident onto the wound during the current pulse are those of nitrogen and oxygen. As such, our idealized conditions are a good approximation.

IEADs to surfaces are computed using the Plasma Chemistry Monte Carlo Module (PCMCM), as described in [21]. The PCMCM is executed on a Cartesian mesh that overlays a subset of the computational domain. Pseudoparticles representing ions are launched from sites within the PCMCM mesh with weightings proportional to their rate of generation by electron impact and heavy particle reactions. 
Pseudo-particles are also launched from the boundaries of the PCMCM mesh in proportion to the entering fluxes of ions. Monte Carlo techniques are used to advance their trajectories in time varying electric fields while accounting for elastic and inelastic collisions. Electric potentials as a function of position computed on the unstructured mesh are interpolated onto the rectilinear structured mesh that overlays the unstructured mesh to enable the Monte Carlo algorithms to more rapidly execute. The energy and angle of particles as they strike surfaces are recorded over a predetermined period of time to provide IEADs as a function of location on the solid surface and time. The typical spatial resolution for the structured PCMCM meshes is $0.3 \mu \mathrm{m}$.

The human skin modelled in this investigation consists of a thin outer layer, the epidermis and an inner layer, the dermis. Four layers of cells are resolved in the epidermis. The wound is a small slice in the skin down to the dermis which exposes live cells to the plasma, as shown in figure 1. One type of wound investigated is dry and shallow, and may represent later stages of wound healing. The other type of wound is filled by a biological fluid. Cellular structures are represented as lossy dielectrics with conductivities and permittivities appropriate for biological tissue. Cell membranes have low conductivity $\left(10^{-7} \Omega^{-1} \mathrm{~cm}^{-1}\right)$; cytoplasm and nucleoplasm have high conductivity $\left(10^{-3}-10^{-4} \Omega^{-1} \mathrm{~cm}^{-1}\right)$. The dielectric constant of the cell membranes, cytoplasm and nucleoplasm are $\varepsilon / \varepsilon_{0}=5.8,30$ and 20, respectively [22,23].

A schematic of the model geometry for DBD treatment of wounded human skin is shown in figure $1(a)$. The wound is in the epidermis of a human thumb which serves as a floating electrode to a DBD. The upper powered electrode positioned over the wound is covered with a $0.8 \mathrm{~mm}$ thick dielectric. The computational domain is $0.8 \times 0.6 \mathrm{~cm}$ and contains the end of the thumb. (See figure 1 of [20].) The sides and bottoms of the computational domain are grounded-the top is powered. The vertical arrows in figure 1(a) show the locations at the upper dielectric from which filaments are launched. The dead skin cells, epidermis and dermis are shown in figure $1(b)$. The smallest wound dimension is of the order of $8 \mu \mathrm{m}$ which corresponds to the single missing cell at the bottom of the wound, and the internal dimensions of the wound are resolved to about $0.5 \mu \mathrm{m}$. The wet wound covered by biological fluid is shown in figure 1(c). The liquid has a dielectric constant of 30. The locations $\mathrm{A}-\mathrm{C}$ in figure $1(b)$ are the sites where IEADs are recorded. The arrows show the local normal which corresponds to an angle of incidence of $0^{\circ}$. The chords along $\mathrm{D}-\mathrm{A}$ in figure $1(b)$ and along $\mathrm{F}-\mathrm{E}$ in figure $1(c)$ indicate the line along which profiles of electric field will be plotted.

\section{Description of the MD model}

The Tersoff-Brenner style reactive empirical bond order (REBO) [24] is employed to describe $\mathrm{C}-\mathrm{H}-\mathrm{O}$ interactions for the MD simulations, and is similar to the one employed and described previously in simulating sputtering of oxygencontaining polymers [25]. One major assumption is the neglect of long-range van der Waals interactions for reasons described

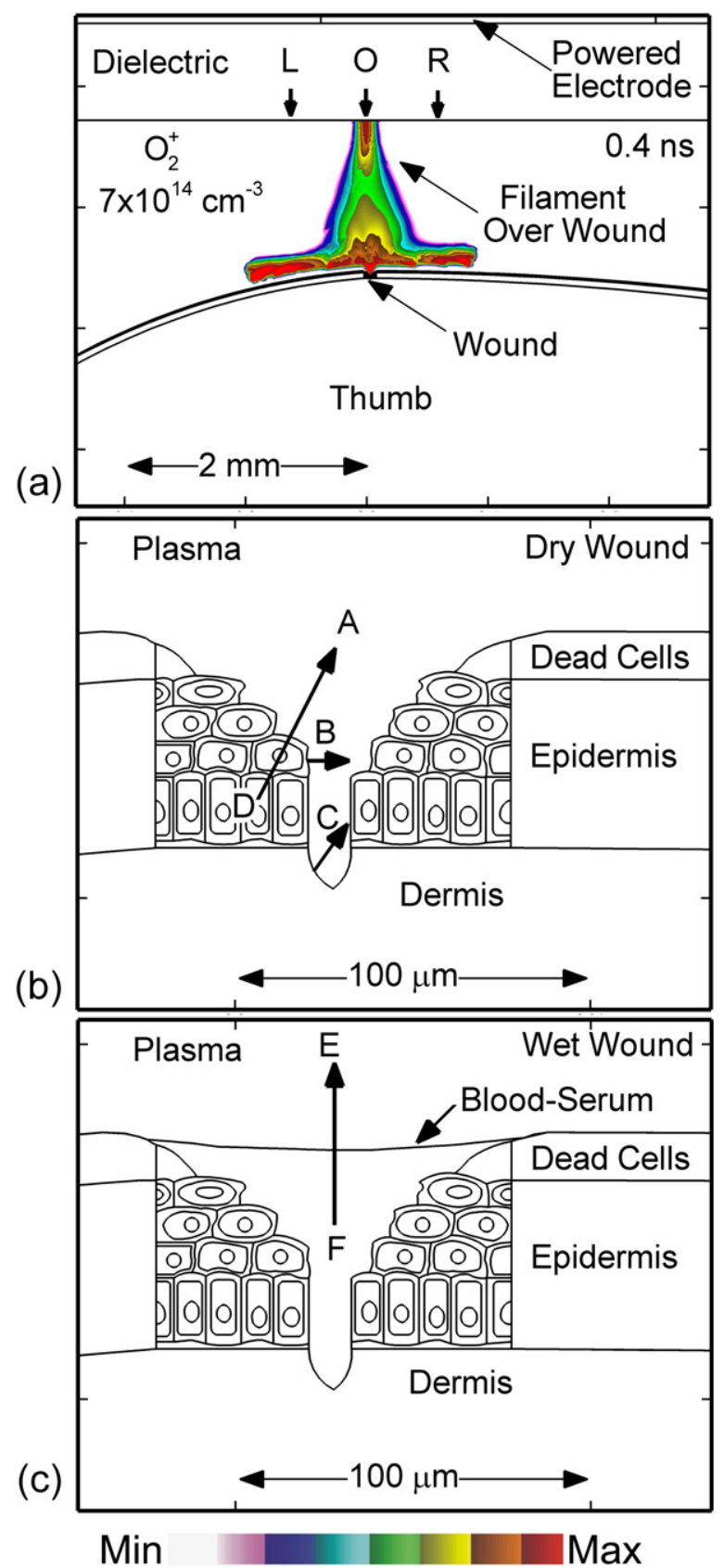

Figure 1. Schematic of the model geometry for DBD treatment of wounded human skin. (a) Typical plasma filament sustained in atmospheric pressure air incident onto a thumb with a small wound The upper electrode is powered and the thumb serves as a floating electrode. The wound is represented as a small cut in the epidermis layer. The arrows labelled L, O, R show the locations from which filaments to the left, over or right of the wound filaments are launched. The plasma filament over the wound $\left(\mathrm{O}_{2}^{+}\right.$ion density) is shown at $t=0.4 \mathrm{~ns}$. (b) Close-up of the dry wound showing exposed cells, the layer of dead cells, epidermis and dermis. The normal vectors $\mathrm{A}, \mathrm{B}, \mathrm{C}$ show locations where ion energies are recorded. (c) Close-up of wet wound covered by blood serum $\left(\varepsilon / \varepsilon_{0}=30\right)$. Normal vector F-E shows the location where ion energies are recorded.

in detail elsewhere, but briefly, we have found that ionpolymer interactions tend to be dominated by the covalent bond breaking and recombination dynamics that occur in the short times $(\sim 1 \mathrm{ps})$ of the collision cascades. Newton's equations 
of motion are integrated numerically with the velocity Verlet algorithm using a time step of $0.1 \mathrm{fs}$. The Berendsen thermostat is used to control the system temperature. The initial simulation cell consists of sets of 36 vertically aligned acyl chains $(6 \times 6$ arrays $)$ of our model lipid A molecules, following approximately the structure described by Kato et al [26]. The acyl chains consist of $\mathrm{C}_{15} \mathrm{H}_{29} \mathrm{O}_{2}$ and $\mathrm{C}_{15} \mathrm{H}_{28} \mathrm{O}_{3}$ (attached to beta hydroxy group), with the ratio of $2: 1$.

The initial surface is constructed from these 36 chains and is allowed to equilibrate under the simulation procedure for several tens of picoseconds to yield a relaxed surface at $300 \mathrm{~K}$. The dimensions of the simulated film are approximately $2.3 \mathrm{~nm} \times 3.6 \mathrm{~nm}$ and $6.8 \mathrm{~nm}$ deep. The initial density of the film was found to match typical polymers $\left(\sim 0.8 \mathrm{~g} \mathrm{~cm}^{-3}\right)$. The lateral boundaries are treated as periodic and the bottom layer (consisting in this case of entire lipid A chains) is initially held fixed. In order to allow simulations of films deeper than a single C15 chain, we connect these model lipid A segments together and as material is sputtered from the surface, additional chains are added. This is thought to mimic real films with many layers of biopolymer present. We note that similar strategies worked well in simulation of ion sputtering of non-biological polymers [25]. Simulated $\mathrm{Ar}^{+}$ions are initially placed at random positions laterally at a height beyond the cut-off of the potential, and are directed at normal incidence to the surface. Ions are assumed to be neutralized before impact via Auger processes and therefore all species are treated as fast neutrals. A Moliere-type potential is used to model interactions of $\mathrm{Ar}$ with all other species. At the end of each impact lasting between 0.5 and $10 \mathrm{ps}$, any sputtered products and remaining Ar are removed and the cell is cooled to $300 \mathrm{~K}$. The next ion is launched and the process is repeated until results such as average sputtering yield reaches a steady state.

\section{IEADs onto dry and wet wounds}

We launched positive plasma filaments from locations L (left), $\mathrm{O}$ (over) and $\mathrm{R}$ (right) from the upper dielectric. These locations determine the position of the filament to the left, over or to the right of the wound, respectively, as shown in figure $1(a)$. The skin is initially uncharged and the DBD voltage is $40 \mathrm{kV}$. Typical plasma densities are $10^{15} \mathrm{~cm}^{-3}$. When the plasma strikes the surface of the thumb, the surface charges as in a conventional DBD, resulting in a spreading of the plasma along the surface, as shown in figure 1( $a)$. This behaviour is very similar to the interaction of DBDs with organic materials, such as hydrocarbon polymers. The behaviour and spreading of such filaments is discussed in [17].

When the plasma filament is far from the surface, the electric field in the tip of the filament is of the order of $150-200 \mathrm{kV} \mathrm{cm}^{-1}$. When the filament strikes a surface, in this case a cell membrane, thereby bridging the gap with a conductive channel, the majority of the applied voltage is momentarily transferred to the sheath at the surface of the wound. This results in electric fields being many hundreds of $\mathrm{kV} \mathrm{cm}^{-1}$ lasting for a few ns. Given these electric fields, with mean free paths of about one-half micron for ions at atmospheric pressure, one might expect ion energies of up to tens of $\mathrm{eV}$ incident onto the membrane surface produced by acceleration in the sheath. (The time to accelerate an oxygen ion across its mean free path of $0.5 \mu \mathrm{m}$ to an energy of $20 \mathrm{eV}$ in an electric field of $400 \mathrm{kV} \mathrm{cm}^{-1}$ is $<0.1 \mathrm{~ns}$.)

The propagation of the space charge wave in the head of the filament in the vicinity of the wound and the electric field in the wound for the positive filament directly over the wound are shown in figure 2 . The space charge, having densities exceeding $6 \times 10^{15} \mathrm{~cm}^{-3}$, defines the location of the ionization front of the filament. After striking the wound, the filament spreads over the tissue surface with a speed of $5 \times 10^{8} \mathrm{~cm} \mathrm{~s}^{-1}$. With an electron density of nearly $10^{15} \mathrm{~cm}^{-3}$ and electron temperature of $3-5 \mathrm{eV}$, the Debye length $\lambda_{\mathrm{D}}<1 \mu \mathrm{m}$. As a result, the plasma is able to penetrate into the wound. A sheath is formed conformal to the surface of the exposed cells, though not into the narrowest feature at the centre of the wound. As the filament approaches the wound and electric potential is compressed between the head of the filament and the exposed cells, the electric field in the sheath exceeds $700 \mathrm{kV} \mathrm{cm}^{-1}$, after which it begins to dissipate. The thickness of the sheath decreases from $15 \mu \mathrm{m}$ to $7 \mu \mathrm{m}$ as the discharge spreads along the surface.

The time evolution of IEADs for $\mathrm{O}_{2}^{+}$onto the cell surfaces at sites $\mathrm{A}, \mathrm{B}$ and $\mathrm{C}$ is shown in figure 3 . The angle integrated ion energy distributions (IEDs) for location A are shown in figure 4. Normal vectors to these sites are shown in figure $1(b)$. Ions incident onto site A arrive nearly normally to the surface of the cell. Ions incident onto sites B and C initially have grazing angles. As the head of the plasma filament approaches the wound $(t<0.3 \mathrm{~ns}$ ), the potential is expelled from the conductive channel of the filament and compressed in the space ahead of the channel and the surface. Ions incident onto the wound are mostly produced by photoionization ahead of the ionization front and are accelerated in this enhanced field. Ion energies are $0.1-1 \mathrm{eV}$. These energies are commensurate with the effective equilibrium drift temperature of oxygen ions in the vacuum fields [27]. As the ionization front enters into the wound and the sheath is formed at the surface of the cells, the ion energies onto the cells increase. At site A, whose surface normal is nearly vertical, the bulk of the IEAD extends to $15 \mathrm{eV}$ at $0.32 \mathrm{~ns}$ with a tail that extends to nearly $100 \mathrm{eV}$ corresponding to the peak electric field of $>700 \mathrm{kV} \mathrm{cm}^{-1}$. The epithermal ions (energies $\leqslant 1 \mathrm{eV}$ ) have essentially isotropic angles of incidents. At the time when the tail of the IEAD extends to the highest energy ( $0.34 \mathrm{~ns}) 14 \%$ of ions have energies above $2 \mathrm{eV}, 12 \%$ above $5 \mathrm{eV}, 8 \%$ above $10 \mathrm{eV}$ and $5 \%$ above $20 \mathrm{eV}$. The angle of incidence of ions above $1 \mathrm{eV}$ is $\pm 20^{\circ}$. The IEAD begins to degrade in energy as the sheath spreads and the sheath voltage diminishes. However, there is still a substantial tail to the IEAD that extends above $40 \mathrm{eV}$.

The IEADs incident onto site B have nearly the same thermal component as site $\mathrm{A}$ and the ions have nearly the same maximum extent in energy, however, the ions initially arrive at grazing angles. Since the length of the chord for ions to travel through the sheath to arrive at Site B is longer than for site A, the ions are moderately more collisional, and so the thermal component is larger. At the time that the tail of the IEAD extends to its greatest energy, $11 \%$ have energies above $2 \mathrm{eV}$, 


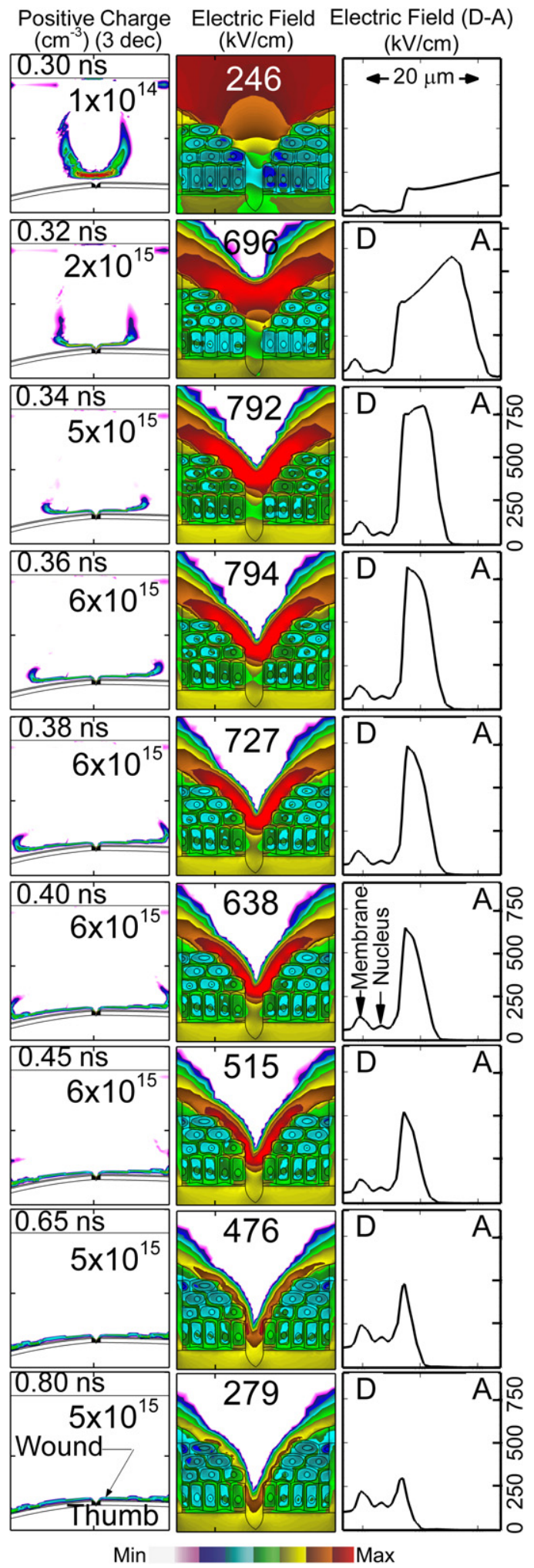

Figure 2. Time sequence of positive space charge (left column) and electric field (centre column) for a positive filament arriving directly over the wound. The electric field along the chord D-A (see figure $1(b)$ ) is also shown (right column). As the plasma filament (conditions: dry air, $+40 \mathrm{kV}$ ) approaches the wound and the applied potential is compressed between the filament head and the cells, the electric field exceeds $700 \mathrm{kV} \mathrm{cm}^{-1}$. (The contours are plotted on a $\log$ scale over 3 decades with the maximum value shown in each frame.)

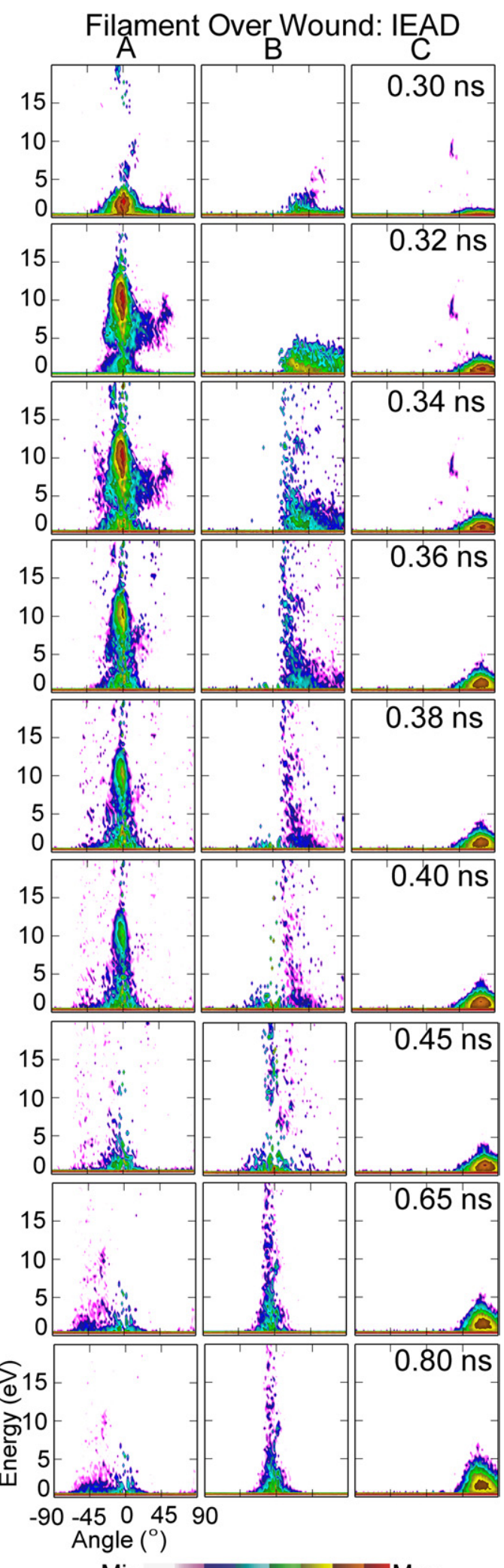

Min $\quad$ Max

Figure 3. IEADs as a function of time for a plasma filament arriving over the wound for locations $\mathrm{A}, \mathrm{B}$ and $\mathrm{C}$ with the normal vectors shown in figure $1(b)$. Ions to site $\mathrm{A}$ arrive almost normally to the cell surface. Ions to site B initially have grazing angles but are later oriented vertically. DBD conditions are dry air and $+40 \mathrm{kV}$. (The contours are plotted on a log scale over 3 decades.) 

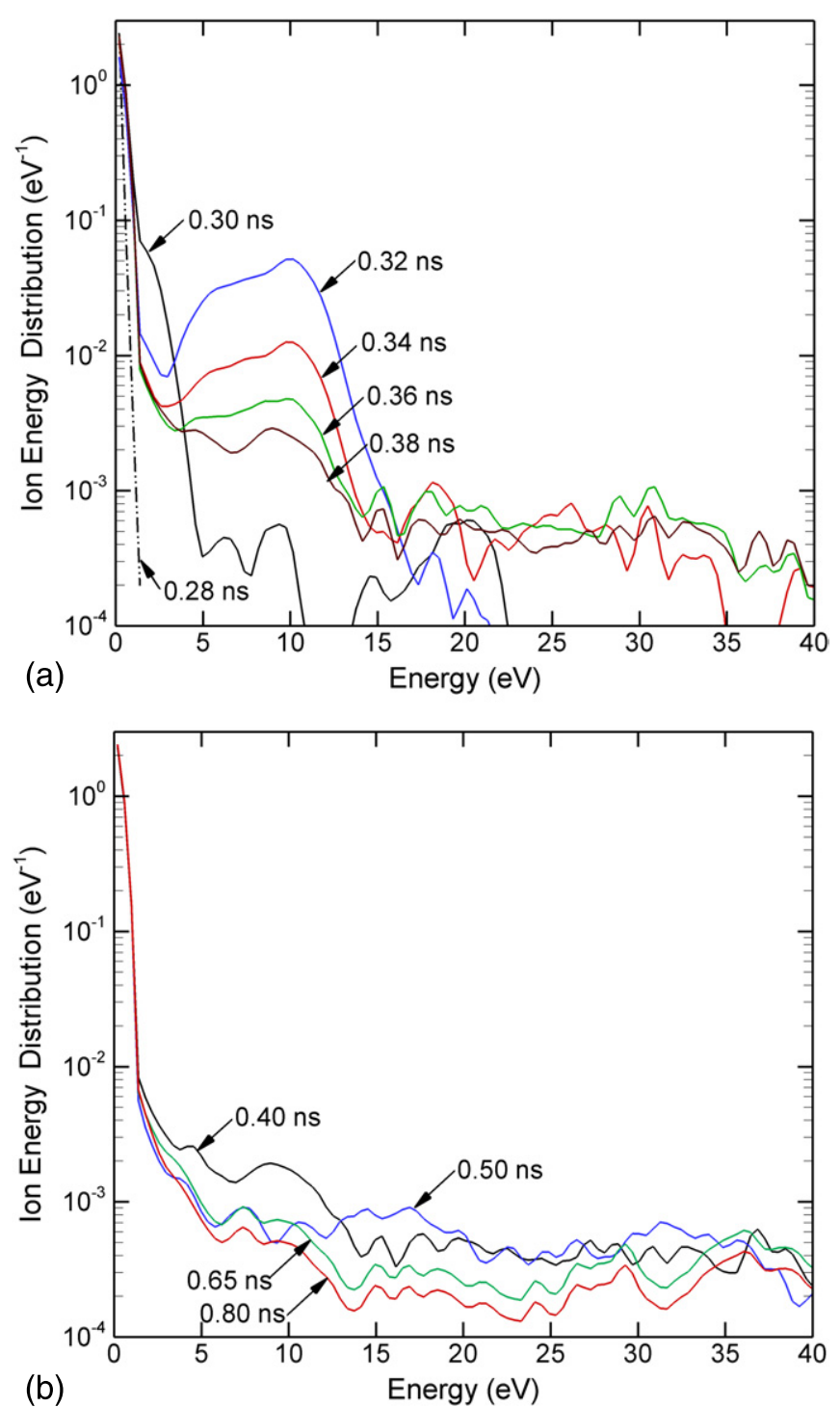

Figure 4. IEDs integrated over angle of incidence onto site $\mathrm{A}$ for $(a)$ times up to $0.38 \mathrm{~ns}$ and $(b)$ times of $0.4 \mathrm{~ns}$ and greater.

$6 \%$ have energies above $10 \mathrm{eV}$ and $4.5 \%$ have energies about $20 \mathrm{eV}$. As the sheath penetrates further into the wound and the plasma density increases, thereby decreasing the thickness of the sheath, the sheath becomes more conformal to the contour of the cell. That is the sheath edge become locally parallel to the surface of the cell. As a result at later times, the IEADs which initially arrive at grazing angles transition to having ions arriving with more normal incidence, as shown for site B in figure 3 .

Due to charging of the cell membranes, site $\mathrm{C}$ sits below the sheath. The Debye length of many microns is still commensurate with the size of the opening and so is not able to conformally cover the cell surfaces at site $\mathrm{C}$. Ions are accelerated through the sheaths to energies commensurate to sites $\mathrm{A}$ and $\mathrm{B}$. However, these ions undergo collisions prior to arriving at the surface. The ions arrive at site $\mathrm{C}$ with grazing angles with significantly lower energies than at sites A and B. At the time the IEAD has its greatest extent, only $2 \%$ of ions have energies greater than $2 \mathrm{eV}$ and only $0.4 \%$ above $10 \mathrm{eV}$.

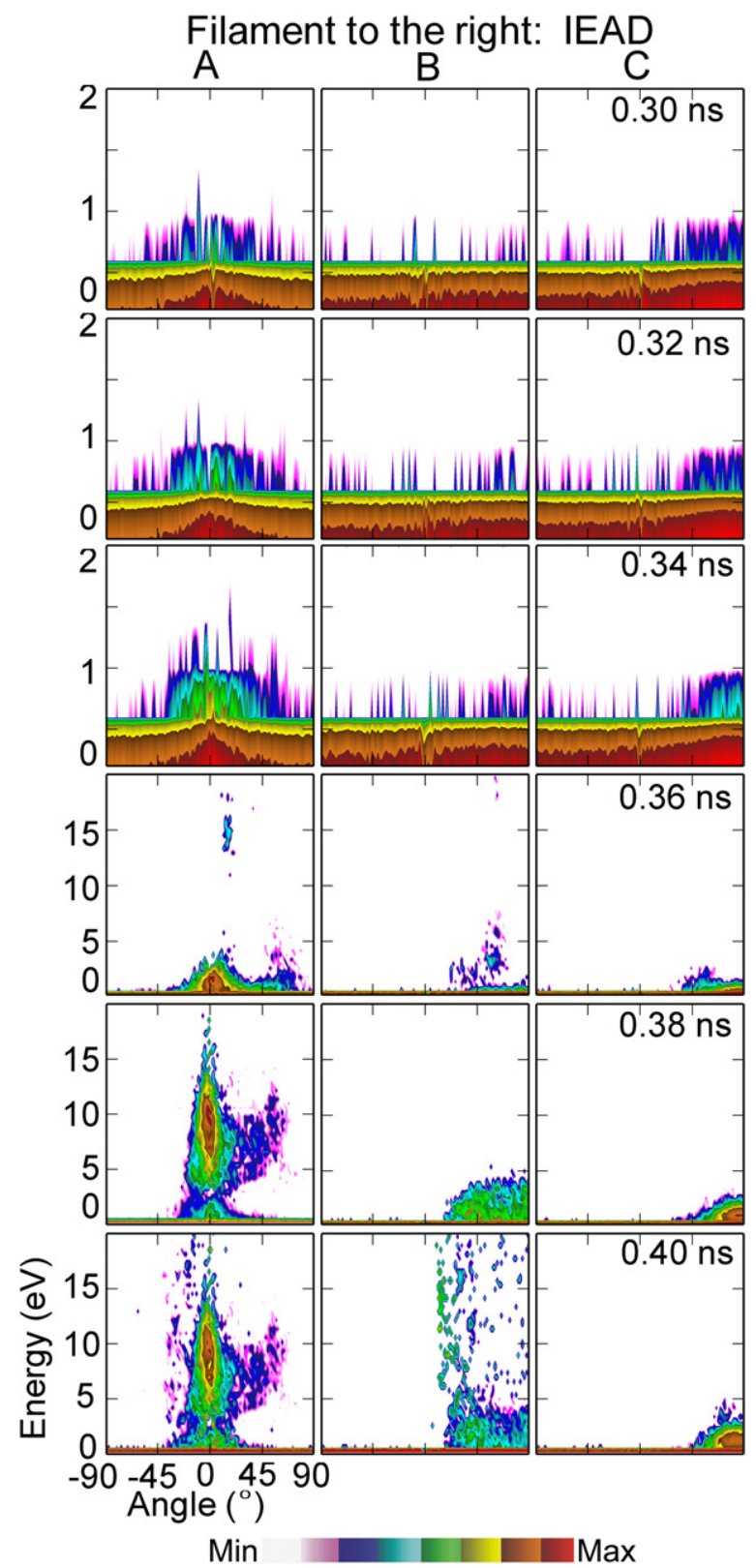

Figure 5. IEADs as a function of time for a plasma filament arriving to the right of the wound. IEADs are shown for locations A, B and $\mathrm{C}$ with the normal vectors shown in figure $1(b)$. DBD conditions are dry air and $+40 \mathrm{kV}$. (The contours are plotted on a log scale over 3 decades.)

When the DBD filament strikes off centre of the wound, energetic ions can still be delivered to cells within the wound. For example, IEADs are shown in figure 5 for a filament striking to the right of the wound and in figure 6 to the left of the wound. The filament strikes the skin surface at approximately $0.3 \mathrm{~ns}$ in each case. As the filament spreads on the surface of the skin approaching the wound, the few ions that strike the wound are largely thermal with energies of $<0.5 \mathrm{eV}$. When the filament reaches the wound the plasma conformally envelopes the surface of the cells, forming a sheath whose voltage drop is essentially the same as for directly striking filament. The IEADs incident onto sites A, B and C therefore largely resemble those for the directly striking filament, but 


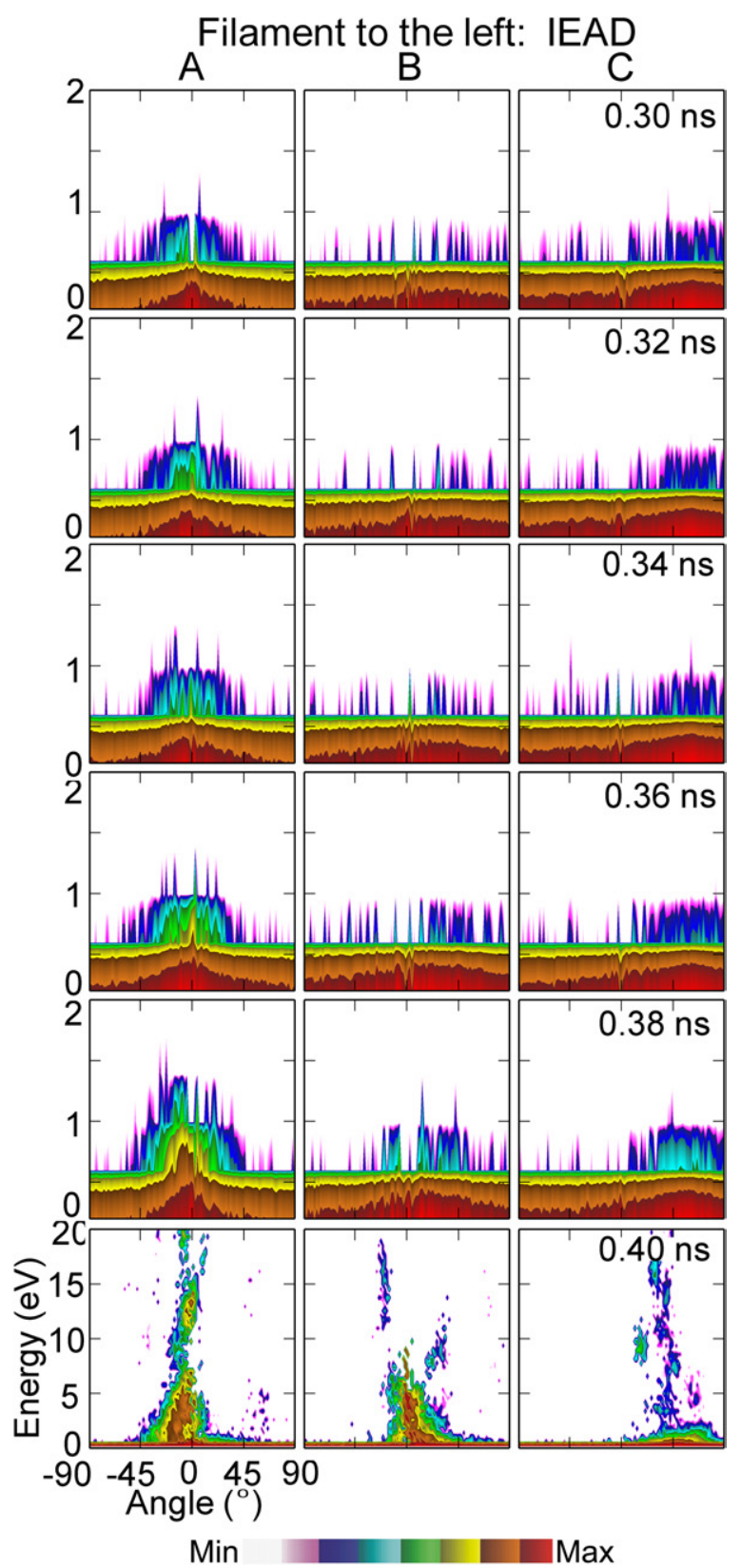

Figure 6. IEADs as a function of time for a plasma filament arriving to the left of the wound. IEADs are shown for locations A, B and C with the normal vectors shown in figure $1(b)$. DBD conditions are dry air and $+40 \mathrm{kV}$. (The contours are plotted on a log scale over 3 decades.)

are delayed in time to account for the spreading time from the original site of impact. For the filament striking to the left of the wound (shown in figure 6), the IEADs are slightly additionally delayed and degraded in energy. This is due to the curvature of the thumb surface. The filament striking to the left of the wound has a larger gap between the electrode and the skin surface, and so a slower propagation speed and lower electric field in the spreading sheath.

It is often the case that wounds are covered with a fluid resembling blood serum which has a large dielectric constant. The plasma filaments then intersect with a liquid interface. The compression of the electric field on the surface of the

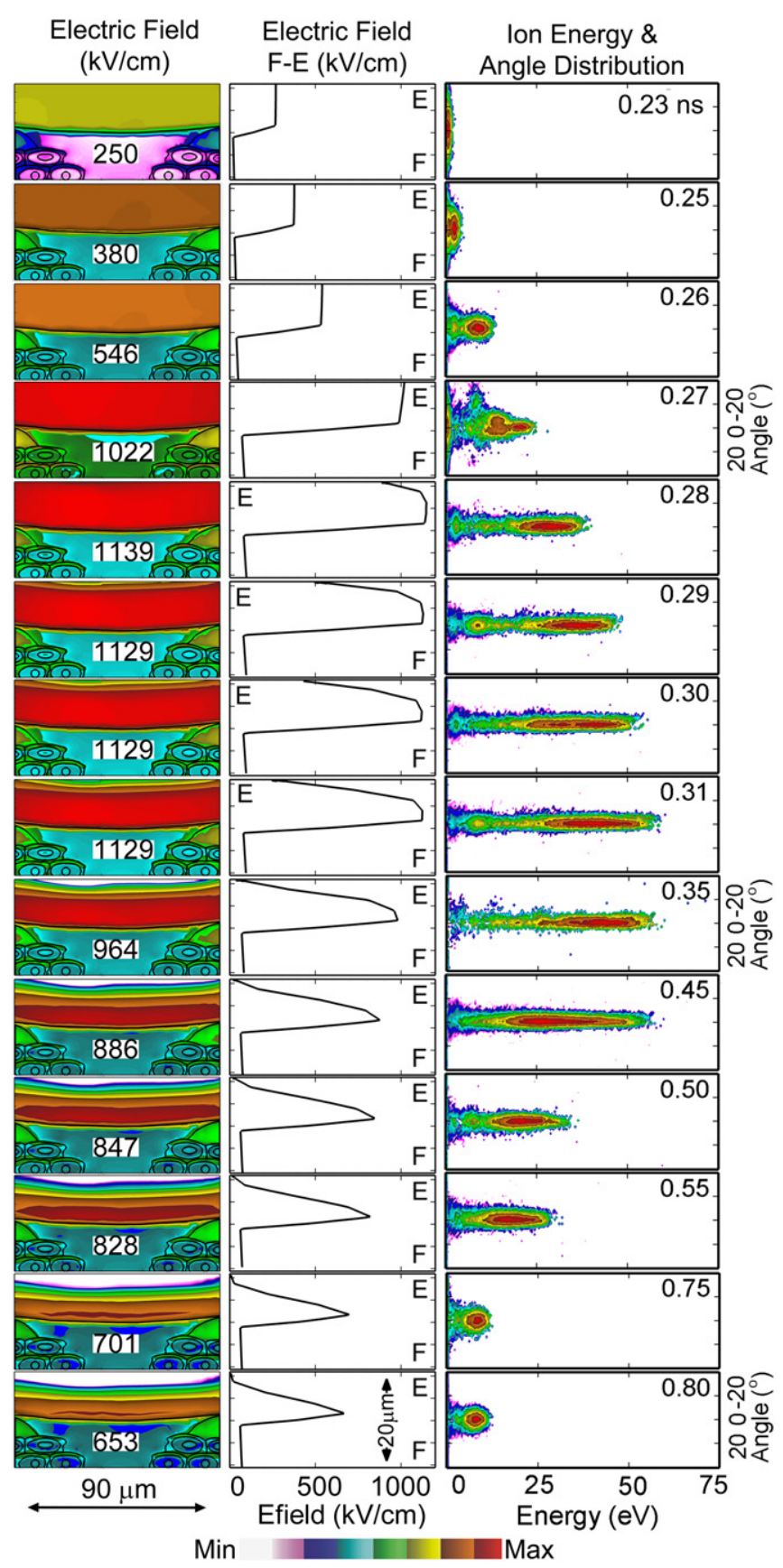

Figure 7. Electrical properties for plasma filament striking the wet wound as a function of time. Left: electric field contours, (centre) electric field along the chord F-E (shown in figure 1(c)) and (right) IEAD striking the top surface of the liquid. DBD conditions are dry air and $+40 \mathrm{kV}$. (The contours are plotted on a log scale over 3 decades.)

liquid upon arrival of the streamer, and IEADs delivered to the liquid-gas interface for a wound filled with liquid having $\varepsilon / \varepsilon_{0}=30$ are shown in figure 7 . The angle integrated IEDs are shown in figure 8 . As in the case of the dry wound, ions are thermalized prior to arrival of the filament onto the liquid surface. As the sheath forms and dissipates, a pulse of energetic ions with energies exceeding $60 \mathrm{eV}$ is delivered to the surface. The maximum ion energies increase as the sheath is formed and the electric field in the sheath increases. The peak electric field in the sheath momentarily reaches in excess of $900 \mathrm{kV} \mathrm{cm}^{-1}$. 

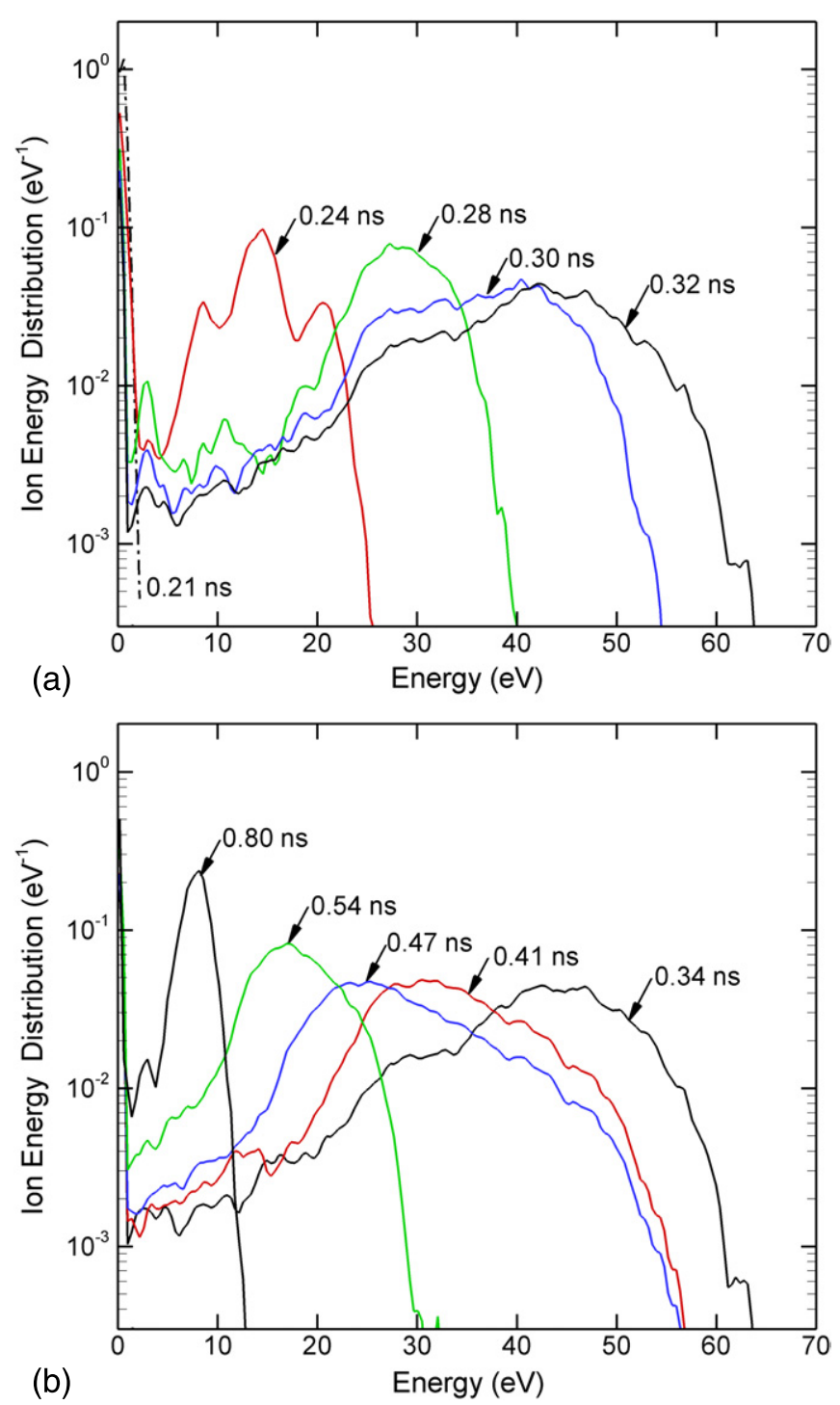

Figure 8. IEDs integrated over angle of incidence onto the surface of the liquid for $(a)$ times up to $0.32 \mathrm{~ns}$ and $(b)$ times of $0.34 \mathrm{~ns}$ and greater.

The IEDs decrease in energy as the sheath dissipates when the plasma spreads on the surface of the thumb outside the wound. At the time when the tail of the IED extends to the highest energy $(0.34 \mathrm{~ns}) 88 \%$ of ions have energies above $20 \mathrm{eV}, 82 \%$ above $30 \mathrm{eV}, 55 \%$ above $40 \mathrm{eV}$ and $16 \%$ above $50 \mathrm{eV}$.

The IEADs for ions arriving onto the liquid interface extend to higher energies than IEADs incident onto the cells in the dry wound. This observation is consistent with the scaling of IEADs discussed in [17] where ions arriving onto dielectric surfaces with larger $\varepsilon / \varepsilon_{0}$ have higher energies. This scaling results from electric field lines being expelled from the large $\varepsilon / \varepsilon_{0}$ material (or a longer time is required to charge the higher capacitance of the large $\varepsilon / \varepsilon_{0}$ material), thereby leaving more voltage to be expended in the sheath. The IEADs onto the liquid also have a smaller range in angles, reflecting the fact that the intersection of the filament with the wound is largely parallel to the normal to the surface.

The dielectric relaxation time of cellular material is as large as $5 \mu \mathrm{s}$ for the cell membranes and $<1 \mathrm{~ns}$ in the more conductive cytoplasma. Since the rise-time of the electric field arriving onto the cells is a few tenths of a ns, one might expect significant electric field penetration into the cells. The charge density and magnitudes of the electric field inside the cells for the dry wound and the wound covered with blood serum are shown in figure 9. The maximum electric field at the surface of the bare cells is in excess of $700 \mathrm{kV} \mathrm{cm}^{-1}$. The short duration of the intersection of the filament with the cell membrane is less than or commensurate with the dielectric relaxation time of the underlying cellular material, and so there is penetration of the electric field into the cells. Peak electric fields of up to $140 \mathrm{kV} \mathrm{cm}^{-1}$ across cell membranes and $80 \mathrm{kV} \mathrm{cm}^{-1}$ across the nucleus are predicted. (See also figure 2.) Due to the intervening dielectric properties of the liquid, the electric fields that penetrate into the cells below the blood serum are smaller than the dry wound, but not significantly so. Electric fields across the cell membranes are $120 \mathrm{kV} \mathrm{cm}^{-1}$ and across the nucleus are $70 \mathrm{kV} \mathrm{cm}^{-1}$. As discussed in [20], the voltage drop across the thickness of an actual lipid membrane $(5-10 \mathrm{~nm})$ is about $0.1 \mathrm{~V}$, a value corresponding to the lower limit of cell electroporation for long pulses. This is not a large enough value to physically disrupt the cell. As DBDs consist of numerous filaments, the cumulative effect of multiple filaments may lead to significant electroporation. Note that we do not resolve the nuclear membranes.

\section{MD simulation of ion bombardment of model lipid-like films}

Typical sputtering results from the MD simulations of model lipid-like materials are shown in figure 10. The averaged carbon (C) sputter yields for $\mathrm{Ar}^{+}$ion impact at normal incidence as a function of ion energy is in figure $10(a)$ and side views of the layers before (left) ion impact and after (right) for an ion fluence of $6 \times 10^{16} \mathrm{~cm}^{-2}$ are shown in figure $10(b)$. The sputtering threshold energy appears to be between 10 and $20 \mathrm{eV}$. At an ion energy of $50 \mathrm{eV}$, the yield rises to $\sim 3-4$ and increases to $\sim 10$ at $100 \mathrm{eV}$. Unlike the case of other polymers (e.g., PMMA or polystyrene), these model lipid-like materials do not show the formation of a thin C-rich layer near the surface that is known to significantly reduce ion sputtering yields. For example, polystyrene films impacted at normal incidence by $150 \mathrm{eV} \mathrm{Ar}^{+}$show an initial $\mathrm{C}$ sputter yield of $\sim 5$, but after an ion fluence of $\sim 10^{16} \mathrm{~cm}^{-2}$, the yield drops by about 2 orders of magnitude. It appears that these model lipid-like materials contain too much $\mathrm{O}$ and $\mathrm{H}$ to allow this transition to occur. Choudhary et al [25] reported a similar result for polyethylene and Teflon model polymer films subject to ion bombardment. Although not shown explicitly here, simulations of room temperature $\mathrm{O}$ atom impacts of these model films show that spontaneous etching can occur.

\section{Implications for plasma treatment of exposed cell membranes}

When treating tissue and biological liquids with DBDs, represented here by small dry and wet wounds in skin, transient sheaths of hundreds of $\mathrm{kV} \mathrm{cm}^{-1}$ are produced at their surfaces. 


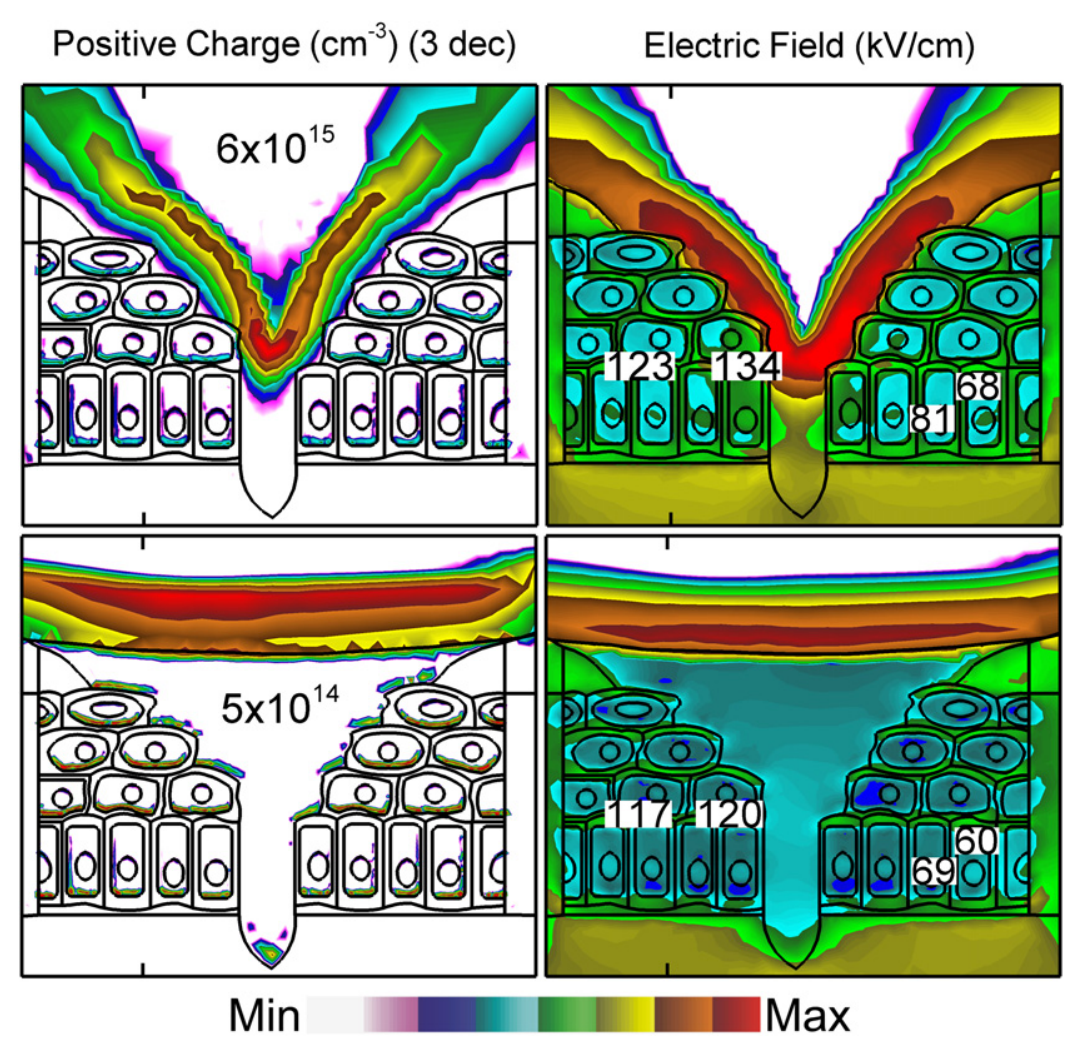

Figure 9. Positive charges and electric fields for (top row) dry wound and (bottom row) wet wound.

Even at atmospheric pressure, ions that are produced near to cell membranes by these plasma filaments can be accelerated to energies of tens of $\mathrm{eV}$. The character of the IEAD delivered to the cell membranes depends on the location of the plasma filament with respect to the wound and orientation to the filament to the cell surface. Nevertheless, even deep in the wound incident ion energies are up to $5 \mathrm{eV}$. The energies of ions incident onto the surface of biological liquids tend to be higher (up to $50-100 \mathrm{eV}$ ) due to the larger $\varepsilon / \varepsilon_{0}$ of those materials.

The consequences of low-energy ion bombardment onto biological tissues and liquids are poorly known. In the context of most studies of ion interactions with biological materials, low-energy ion bombardment encompasses energies of many $\mathrm{keV}$ [16]. Although programs such as SRIM [28] are intended for ion energies of many $\mathrm{keV}$, some indication of the potential impact of low-energy ions on biological tissue can be obtained. For example, from SRIM the range of $50-100 \mathrm{eV}$ oxygen ions into material with the atomic composition of human skin is about 1.3 to $1.8 \mathrm{~nm}$. Since the thickness of a typical mammalian cell wall is $10-20 \mathrm{~nm}$, it is not likely that these ion will penetrate the cell wall-but these ions may sputter the cell membrane and activate surface reactions.

If we make analogy to inorganic materials processing, ion energies of tens of $\mathrm{eV}$ are sufficient to activate surface processes, break bonds and sputter atoms on polymer surfaces [29]. In studies of low-energy ion bombardment of polystyrene, $50 \mathrm{eV}$ argon ions produced an ion-damaged layer of $\sim 1.0 \mathrm{~nm}$ [30]. As noted above, MD simulations of $\sim 100 \mathrm{eV} \mathrm{Ar}{ }^{+}$bombardment of polystyrene showed formation of a hydrogen depleted, cross-linked layer 1-2 nm deep [31].
Although there are certainly critical differences between cell walls and such hydrocarbon polymers, these results indicate that cell walls are likely influenced in meaningful ways by ion energies up to tens of $\mathrm{eV}$. The higher ion energies obtained for DBD interactions with liquids make these interactions even more likely. For example, human blood serum is approximately $92 \%$ water and $8 \%$ proteins. These proteins are basically hydrocarbons that likely undergo similar ionmolecule reactions as conventional hydrocarbon polymer materials.

The results from the MD simulations for $\mathrm{Ar}^{+}$ bombardment of lipid layers, with and without $\mathrm{O}$ atoms, is particularly revealing of the potential interactions of the ion and radicals produced in DBDs with materials typical of cell membranes. For example, the results from the MD simulation shown in figure 10(a) indicate carbon yields for sputtering by $\mathrm{Ar}^{+}$increases from the threshold energy of $15 \mathrm{eV}$ to $\sim 0.4$ at $20 \mathrm{eV}$ and to $1-10$ for energies of $35-100 \mathrm{eV}$. These values are for lipid-like films that have already seen an argon ion fluence of $3.8 \times 10^{16} \mathrm{~cm}^{-2}$. Our MD simulations also show that in the presence of an atomic oxygen flux with an ion/neutral ratio of $1: 10$, the carbon sputtering yield is more than 0.1 at ion energies as low as $20 \mathrm{eV}$.

To estimate the consequences of DBD plasma treatment of cell membranes, we assumed that the carbon sputtering yields by $\mathrm{Ar}^{+}$produced by the MD simulation are representative of ions having similar masses, such as $\mathrm{O}_{2}^{+}$. This assumption is likely a lower limit to the sputtering yield given the more reactive nature of the oxygen and nitrogen ions produced in air DBDs. We can then convolve these energy-dependent 


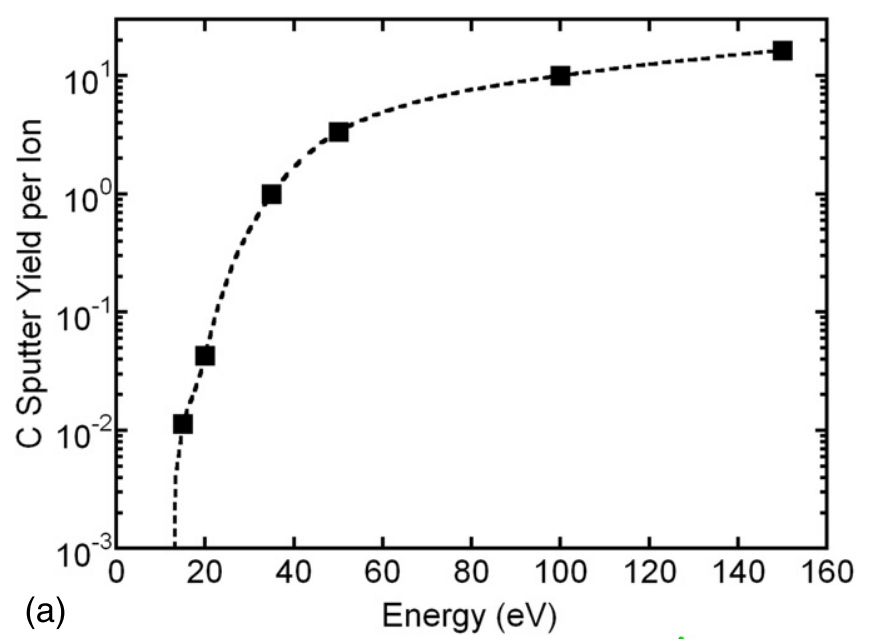

(a)

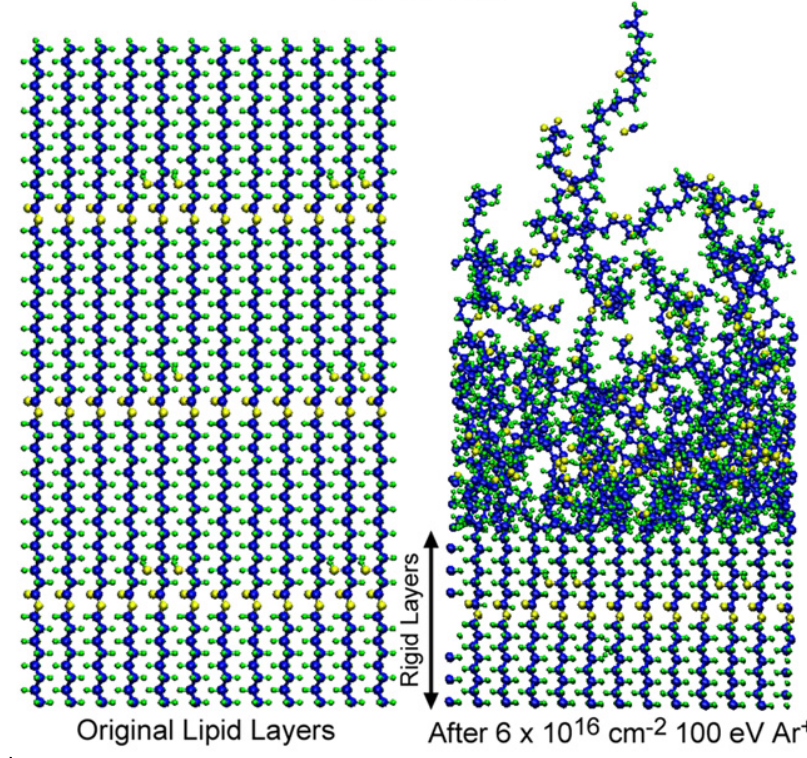

(b)

Figure 10. Results of MD simulation for sputter yield of carbon atoms from the lipid-like layer resulting from $\mathrm{Ar}^{+}$bombardment. (a) Carbon yield per $\mathrm{Ar}^{+}$for an ion fluence of $3.8 \times 10^{16} \mathrm{~cm}^{-2}$. (b) Simulated lipid-like layer (left) before ion bombardment and (right) after bombardment of $100 \mathrm{eV}$ ions with fluence of $6 \times 10^{16} \mathrm{~cm}^{-2}$.

sputtering yields shown in figure $10(a), Y(\varepsilon)$, with the timedependent IEDs, $f(\varepsilon, t)$, to produce an effective, IED averaged sputtering yield, $Y_{\mathrm{eff}}(t)$ per ion at a particular time,

$$
Y_{\mathrm{eff}}(t)=\int f(\varepsilon, t) Y(\varepsilon) \mathrm{d} \varepsilon .
$$

By convolving $Y(\varepsilon)$ with the ion fluxes, $\phi(t)$, over the duration of the pulse and dividing by the area density of sites, $\rho$, we can estimate the carbon atom removal per site, $N_{c}$ per pulse

$$
N_{c}=\frac{1}{\rho} \iint \phi(t) f(\varepsilon, t) Y(\varepsilon) \mathrm{d} \varepsilon \mathrm{d} t .
$$

Multiplying $N_{c}$ by pulse repetition rate $R$, produces the time rate of removal of $\mathrm{C}$ atoms per site.

$Y_{\text {eff }}(t)$ for ions incident on site A as a function of time during the arrival of the plasma filament onto the cell is shown in figure 11. The peak value of the sputtering yield $Y_{\text {eff }}(t)$ is about 0.2 though this occurs prior to the arrival of the peak of

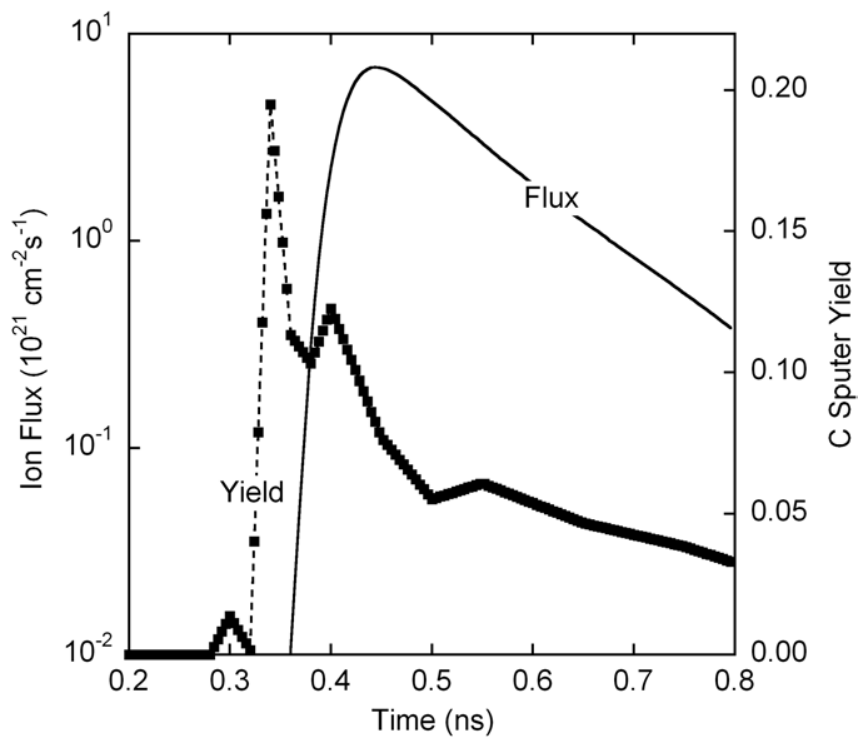

Figure 11. Total ion flux and carbon atom sputter yield as a function of time during a single DBD pulse onto site A. The yield is obtained by convolving the ion energy-dependent yield obtained from the MD simulations with the IEADs obtained from the plasma simulation.

the ion pulse. Upon arrival of the main part of the ion flux, approaching $10^{22} \mathrm{~cm}^{-2} \mathrm{~s}^{-1}$, the $Y_{\text {eff }}(t)$ has decreased to about 0.1 as the IEAD relaxes to lower energies. $Y_{\text {eff }}(t)$ asymptotes to essentially zero by $2-3 \mathrm{~ns}$.

The total ion fluence per pulse is about $1 \times 10^{12} \mathrm{~cm}^{-2}$. For a repetition rate of $R=10 \mathrm{kHz}$, typical of DBDs used in plasma medicine applications, $3 \mathrm{~s}$ of exposure to the plasma is required to produce an ion fluence of $3 \times 10^{16} \mathrm{~cm}^{-2}$. To predict pulsed averaged sputter yields, we used a site density of $\rho=5 \times 10^{14} \mathrm{~cm}^{-2}$, and convolved the predicted IEADs and ion fluxes with the sputter yields from the MD simulations as described above. The predicted sputter yield per pulse is $N_{c}=10^{-4}$. For a repetition rate of $10 \mathrm{kHz}$, the total sputter yield is $N_{c} R \approx 1 \mathrm{~s}^{-1}$. That is, the likelihood of removing the average carbon atom from the lipid layer based solely on ion bombardment produced by the DBD is 1.0 for every second of plasma exposure. In addition to the removal of $\mathrm{C}$ atoms, the atoms remaining in the upper echelons of the model lipid layer are significantly spatially mixed from their initial configuration, as shown in figure $10(b)$. This mixing likely affects the biological function of the cell membrane.

When including the additional reactivity of oxygen radicals, significantly more carbon removal may take place. The results of the MD simulation indicate the carbon etching yield of a model lipid layer having already experienced $6 \times$ $10^{15} \mathrm{~cm}^{-2}$ of ion bombardment is about 0.1 . The oxygen fluence during a single discharge pulse is $8 \times 10^{14} \mathrm{~cm}^{-2}$, which produced a rate of carbon removal per site per discharge pulse of about 0.16 , and significant carbon removal on a pulseperiodic basis.

\section{Concluding remarks}

Atmospheric pressure DBDs being investigated for treating human tissue are capable of producing ns pulses of electric 
fields up to many hundreds of $\mathrm{kV} \mathrm{cm}^{-1}$. Even with mean free paths of less than a micron, this produces ion energies incident onto cell membranes of many tens of $\mathrm{eV}$. In the case of DBD treatment of high permittivity biological fluids, the ion energies may exceed $60-70 \mathrm{eV}$. The angle of incidence of the ions initially depends on the orientation of the cell surface to the plasma filament. Since the Debye length of the plasma filament produced by DBDs is commensurate with the contours of exposed cells, the transient sheath is able to conformally cover exposed cells. Due to this conformal covering of the cells, the IEADs may initially arrive at grazing angles but eventually become nearly normal to the surface. Note that there is strong dependence of ion energy on the applied voltage. As the filament is highly conductive, the potential drop along the length of the filament is small and so a large fraction of the applied voltage is dropped in front of the head of the filament. This voltage appears, at least transiently, across the sheath which increases ion energies to the surface and so increases sputtering rates. Although this dependence was not specifically investigated here, the dependence of ion energies distributions delivered by DBDs to flat polymeric surfaces while varying voltage is discussed in [17].

MD simulations of the interaction of ions with model lipid-like films as found on cell membranes predict sputtering yields of many tens of percent to above unity for ion energies produced by atmospheric pressure DBDs. When convolving these sputter yields with the IEDs and operating conditions expected in DBDs used in plasma medicine applications, ion sputter yields of about $1 \mathrm{~s}^{-1}$ per site, or a monolayer every few seconds of plasma exposure, are predicted in addition to significant mixing of the atoms near the top of the lipid-like layer. These yields are significantly higher with a simultaneous flux of oxidizing radicals.

There are additional effects of ions on cell membranes beyond that of physical sputtering. As mentioned above, ion fluxes contribute to charging of cell membranes, production of electric fields interior to the cell and instigation of processes such as electroporation. There is also a heat loading on the cells due to the recombination energy of the incident ion fluxes. Commenting on the therapeutic or biocidal consequences of these effects, and of the predicted sputtering yields, on cells, is beyond the scope of this paper. However, our results do clearly indicate that the ions produced by atmospheric pressure DBDs are not likely to be passive participants in plasma medicine applications.

\section{Acknowledgment}

This work was supported by the US Department of Energy Office of Fusion Energy Science Contract (DE-SC0001939, and DE-SC0002588).

\section{References}

[1] Kong M G, Kroesen G, Morfill G, Nosenko T, Shimizu T, van Dijk J and Zimmermann J L 2009 Plasma medicine: an introductory review New J. Phys. 11115012

[2] Morfill G E, Kong M G and Zimmermann J L 2009 Focus on Plasma Medicine New J. Phys. 11115011
[3] Fridman G, Shekhter A B, Vasilets V N, Friedman G, Gutsol A and Fridman A 2008 Applied plasma medicine Plasma Process. Polym. 5503

[4] Stoffels E 2006 Guest Editorial for Special Issue on 'Gas plasmas in biology and medicine' J. Phys. D: Appl. Phys. 39 doi:10.1088/0022-3727/39/16/E01

[5] Laroussi M, Mendis D A and Rosenberg M 2003 Plasma interaction with microbes New J. Phys. 541

[6] Sladek R E J, Stoffels E, Walraven R, Tielbeek R J A and Koolhoven R A 2004 Plasma treatment of dental cavities: a feasibility study IEEE Trans. Plasma Sci. 321540

[7] Fridman G, Peddinghaus M, Ayan H, Fridman A, Balasubramanian M, Gutsol A, Brooks A and Friedman G 2006 Blood coagulation and living tissue sterilization by floating-electrode dielectric barrier discharge in air Plasma Chem. Plasma Process. 26425

[8] Reshetov I V, Kabisov R K, Shekhter A B, Pekshev A V and Maneilova M V 2000 The use of a 'Plason' air-plasma apparatus for coagulation and NO-therapy in plastic reconstructive surgery for oncologic patients Ann. Plastic, Reconstr. Aesthetic Surg. 424

[9] Shekhte A B, Serezhenkov V A, Rudenko T G, Pekshev A V and Vanin A F 2005 Beneficial effect of gaseous nitric oxide on the healing of skin wounds Nitric Oxide-Biol. Chem. 12210

[10] Kim G C, Kim G J, Park S R, Jeon S M, Seo H J, Iza F and Lee J K 2009 Air plasma coupled with antibody-conjugated nanoparticles: a new weapon against cancer J. Phys. $D$ : Appl. Phys. 42032005

[11] Heinlin J, Morfill G, Landthaler M, Stolz W, Isbary G, Zimmermann J L, Shimizu T and Karrer S 2010 Plasma medicine: Possible applications in dermatology J. Germ. Soc. Dermatol. 8 968-76

[12] Dobrynin D, Fridman G, Friedman G and Fridman A 2009 Physical and Biological mechanisms of direct plasma interaction with living tissue New J. Phys. 11115020

[13] Nosenko T, Shimizu T and Morfill G E 2009 Designing plasmas for chronic wound disinfection New J. Phys. 11115013

[14] Boudam M K, Moisan M, Saoudi B, Popovici C, Gherardi N and Massines F 2006 Bacterial spore inactivation by atmospheric-pressure plasmas in the presence or absence of UV photons as obtained with the same gas mixture J. Phys. D: Appl. Phys. 393494

[15] Kong M G, Keidar M and Ostrikov K 2011 Plasmas meet nanoparticles-where synergies can advance the frontier of medicine J. Phys. D: Appl. Phys. 44174018

[16] Ptasinska S, Mason N J, Hunniford C A, McCullough R W, Denifl S, Mauracher A and Scheier P 2010 Desorption of small ionic fragments from oligonucleotides induced by low energy carbon ions Eur. J. Phys. D 6059

[17] Babaeva N Yu and Kushner M J 2011 Ion Energy and angular distributions onto polymer surfaces delivered by dielectric barrier discharge filaments in air: I. Flat surfaces Plasma Sources Sci. Technol. 20035017

[18] Kylian O, Hasiwa M, Gilliland D and Rossi F 2008 Experimental Study of the Influence of $\mathrm{Ar} / \mathrm{H}_{2}$ microwave discharges on lipid A Plasma Proc. Polym. 5 26-32

[19] Standaert T E F M, Schaepkens M, Rueger N R, Sebel P G M, Oehrlein G S and Cook J M 1998 High density fluorocarbon etching of silicon in an inductively coupled plasma: mechanism of etching through a thick steady state fluorocarbon layer J. Vac. Sci. Technol. A 16239

[20] Babaeva N Yu and Kushner M J 2010 Intracellular electric fields produced by dielectric barrier discharge treatment of skin J. Phys. D: Appl. Phys. 43185206

[21] Agarwal A and Mark J Kushner 2005 Effect of nonsinusoidal bias waveforms on ion energy distributions and fluorocarbon plasma etch selectivity J. Vac. Sci. Technol. A 231440 
[22] Feldman Y, Ermolina I and Hayashi Y 2003 Time domain dielectric spectroscopy study of biological systems IEEE Trans. Dielectr. Electr. Insul. 10728

[23] Ermolina I, Polevaya Y, Feldman Y, Ginzburg B and Schlesinger M 2001 Study of normal and malignant white blood cells by time domain dielectric spectroscopy IEEE Trans. Dielectr. Electr. Insul. 8253

[24] Ni B, Lee K H and Sinnott S B 2004 A reactive empirical bond order (REBO) potential for hydrocarbon-oxygen interactions J. Phys.: Condens. Matter 167261

[25] Choudhary G K, Vegh J J and Graves D B 2009 Molecular dynamics simulations of oxygen-containing polymer sputtering and the Ohnishi parameter J. Appl. Phys. D: Appl. Phys. 42242001

[26] Kato N, Sugiyama T, Naito S, Arakawa Y, Ito H, Kido N, Ohta M and Sasaki K 2000 Molecular structure of bacterial endotoxin (Escherichia coli Re lipopolysaccharide): implications for formation of a novel heterogeneous lattice structure Mol. Microbiol. 36796
[27] Mason E A and Daniel E W 1988 Transport Properties of Ions in Gases (New York: Wiley)

[28] SRIM 2008 (Stopping and Range of Ions in Matter) SRIM-2008, http://www.srim.org/

[29] Oehrlein G S, Phaneuf R J and Graves D B 2011 Plasma-polymer interactions: a review of progress in understanding polymer resist mask durability during plasma etching for nanoscale fabrication J. Vac. Sci. Technol. B 29010801

[30] Bruce R L, Weilnboeck F, Lin T, Phaneuf R J, Oehrlein G S, Long B K, Willson C G, Vegh J J, Nest D and Graves D B 2010 Relationship between nanoscale roughness and ion-damaged layer in argon plasma exposed polystyrene films J. Appl. Phys. 107084310

[31] Végh J J, Nest D, Graves D B, Bruce R L, Engelmann S, Kwon T, Phaneuf R J, Oehrlein G S, Long B K and Willson C G 2008 Molecular dynamics simulations of near-surface modification of polystyrene: bombardment with $\mathrm{Ar}^{+}$and $\mathrm{Ar}^{+} /$radical chemistries J. Appl. Phys. 104034308 